\title{
Antioxidant activity of the giant jellyfish Nemopilema nomurai measured by the oxygen radical absorbance capacity and hydroxyl radical averting capacity methods
}

\author{
KAZUKI HARADA ${ }^{1,6}$, TOSHIMICHI MAEDA ${ }^{1}$, YOSHIRO HASEGAWA ${ }^{3}$, TAKUSHI TOKUNAGA ${ }^{4}$, \\ SHINYA OGAWA ${ }^{2}$, KYOKO FUKUDA ${ }^{5}$, NORIE NAGATSUKA ${ }^{6,7}$, KEIKO NAGAO $^{6,7}$ and SHUNSHIRO UENO ${ }^{1}$ \\ ${ }^{1}$ Course of Resource Management and Food Science, Graduate School of Fisheries Science, and \\ ${ }^{2}$ Laboratory of Marine Food Processing and Safety, National Fisheries University, Independent Administrative Institution, \\ The Ministry of Agriculture Forestry and Fisheries of Japan, Yamaguchi 759-6595; ${ }^{3}$ Owasebussan Co., Ltd., \\ Mie 519-3612; ${ }^{4}$ Otsuya, K.K., Yamaguchi 759-6604; ${ }^{5}$ Okuno Chemical Industries Co., Ltd., Osaka 538-0044; \\ ${ }^{6}$ Research Institute of Domestic Science; ${ }^{7}$ Graduate School of Domestic Science Research, \\ Tokyo Kasei University, Tokyo 173-8602, Japan
}

Received March 28, 2011; Accepted May 18, 2011

DOI: $10.3892 / \mathrm{mmr} .2011 .506$

\begin{abstract}
The giant jellyfish Nemopilema nomurai (reaching sizes of up to $2 \mathrm{~m}$ diameter and $150 \mathrm{~kg}$ ), which forms dense blooms, has caused extensive damage to fisheries by overloading trawl nets, while its toxic nematocysts cause dermatological symptoms. Giant jellyfish are currently discarded on the grounds of pest control. However, the giant jellyfish is considered to be edible and is part of Chinese cuisine. Therefore, we investigated whether any benefits for human health may be derived from consumption of the jellyfish in order to formulate medicated diets. Antioxidant activity of Nemopilema nomurai was measured using the oxygen radical absorbance capacity (ORAC) and hydroxyl radical averting capacity (HORAC) methods. Based on the results, the ORAC value of the giant jellyfish freeze-dried sample was $541 \mu \mathrm{mol}$ trolox equivalent (TE)/100 $\mathrm{g}$ and the HORAC value was 3,687 $\mu$ mol gallic acid equivalent (GAE)/100 g. On the other hand, the $\mathrm{IC}_{50}$ value of hydroxyl radical scavenging activity measured by using the electron spin resonance method was $3.3 \%$. In conclusion, the results suggest that the freeze-dried powder of the giant jellyfish Nemopilema nomurai is a potentially beneficial food for humans.
\end{abstract}

Correspondence to: Dr Kazuki Harada, Course of Resource Management and Food Science, Graduate School of Fisheries Science, National Fisheries University, Independent Administrative Institution, The Ministry of Agriculture Forestry and Fisheries of Japan, 2-7-1 Nagata-Honmachi, Shimonoseki-shi, Yamaguchi 759-6595, Japan

E-mail: kazuki@fish-u.ac.jp

Key words: antioxidant activity, jellyfish, Nemopilema nomurai, oxygen radical absorbance capacity, hydroxyl radical averting capacity

\section{Introduction}

The giant jellyfish, Nemopilema nomurai (Scyphozoa: Rhizostomeae), known as 'echizen kurage' in Japanese, is one of the largest jellyfish in the world's oceans, reaching sizes of up to $2 \mathrm{~m}$ diameter and $150 \mathrm{~kg}$. Blooms of the jellyfish have caused serious damage to net fisheries in the Sea of Japan. Large blooms have been observed in Chinese coastal areas (1), and the cytotoxicity and hemolytic activity have been investigated (2). However, in Dalian, Qingdao and Shanghai the giant jellyfish is considered to be edible and is part of Chinese cuisine. In Japan, the giant jellyfish can be consumed in the restaurant of the Kamo Aquarium in Yamagata Prefecture, while in Tokyo Kasei University, an attempt was made to add freeze-dried giant jellyfish powder to Japanese 'Udon' noodles instead of salt (3). Okazaki performed an analysis of its general constituents and attempted to produce gelatin from Nemopilema nomurai (4).

Previously, we investigated the antioxidant activity of fish sauce (5) and 'Nikogori' gelatin gel food (6) using the alkaline luminol chemiluminescence method. Recently, we reported the antioxidant activity of fish sauce using the oxygen radical absorbance capacity (ORAC) method (7). The ORAC method was developed by Cao et al (8) and improved by the United States Department of Agriculture (USDA) (9). The latest database of ORAC values of foods was disclosed in the homepage of USDA (10).

Furthermore, findings of Schauss et al revealed the antioxidant activity of freeze-dried Amazonian palm berry using ORAC and hydroxyl radical averting capacity (HORAC) methods (11). In addition, Miller et al revealed that a proanthocyanidin-rich Amazonian medical plant has significant chondroprotective and antioxidant actions using ORAC, HORAC and peroxynitrite averting capacity (NORAC) methods. Additionally, these authors found that the plant directly inhibited matrix metalloproteinases and promoted the 
production of the cartilage repair factor, insulin-like growth factor 1 (IGF-1) (12). In this study, in addition to the ORAC and electron spin resonance (ESR) methods $(6,7,13)$, we investigated the benefits for human health using the HORAC method (14) in order to assess the potential of formulating a medicated diet from the freeze-dried powder of Nemopilema nomurai.

\section{Materials and methods}

Nemopilema nomurai samples. Nemopilema nomurai samples were extracted from the Sea of Japan off the coast of Tsuruoka, a city in Yamagata Prefecture, Japan, in November 2007. The sample was freeze-dried using vacuum freeze-drying equipment (SF-02 freeze cabin, Okawara Corporation, Shizuoka, Japan). Another raw edible commercial sample of giant jellyfish from the Bahraini waters, similar to the Nemopilema nomurai, which had been treated with $3 \% \mathrm{NaCl}$ and sodium hydrosulfite as a bleaching agent, was provided to us by Kyushu Maruichi Foods Co., Ltd., Japan.

Chemicals. 2,2'-Azobis (2-amidinopropane) dihydrochloride (AAPH), dipotassium hydrogenphosphate $\left(\mathrm{K}_{2} \mathrm{HPO}_{4}\right)$, cobalt (II) chloride hexahydrate, picolinic acid, gallic acid monohydrate and hydrogen peroxide $\left(\mathrm{H}_{2} \mathrm{O}_{2}\right)$ were obtained from Wako Pure Chemical, Ltd. (Osaka, Japan). Potassium dihydrogenphosphate $\left(\mathrm{KH}_{2} \mathrm{PO}_{4}\right)$ was obtained from Kanto Chemical Co., Inc. (Tokyo, Japan). Fluorescein sodium salt and iron (II) sulfate heptahydrate $\left(\mathrm{FeSO}_{4} \cdot 7 \mathrm{H}_{2} \mathrm{O}\right)$ were purchased from Sigma-Aldrich Japan (Tokyo, Japan). The spin trapping reagent, 5,5-dimethyl-1-pyrroline $\mathrm{N}$-oxide (DMPO) and 6-hydroxy2,5,7,8-tetramethyl-chroman-2-carboxylic acid (trolox) were purchased from Tokyo Kasei Kogyo Co., Ltd. (Tokyo, Japan).

Samplepreparation.Forthe ORACandHORACmeasurements, $2 \mathrm{~g}$ each of the freeze-dried and commercial samples of Nemopilema nomurai were homogenized with $20 \mathrm{ml}$ distilled water using a polytron PT2100 (Kinematica AG, Luzern, Switzerland). The solutions were centrifuged at $2,580 \mathrm{x}$ g for $10 \mathrm{~min}$, and the supernatant was frozen as the stock sample solution. For the ESR measurement, $2 \mathrm{~g}$ freeze-dried and commercial samples were each homogenized using $10 \mathrm{ml}$ distilled water. Treatment procedures of the sample solutions were prepared in the same manner as described above.

Preparation of hydrophilic-oxygen radical absorbance capacity reaction solution. Hydrophilic-ORAC reaction solution was prepared as previously described (7).

Measurement of hydrophilic-ORAC. The experimental procedure of the hydrophilic-ORAC method was performed as previously described (7).

Preparation of HORAC reaction solution. Phosphate buffer was utilized as the assay (control) buffer and was prepared by combining $75 \mathrm{mM} \mathrm{KH}_{2} \mathrm{PO}_{4}$ and $75 \mathrm{mM} \mathrm{KH}_{2} \mathrm{PO}_{4}$, adjusted to $\mathrm{pH}$ 7.4. Fluorescein working solution was prepared at a concentration of $6.7 \times 10^{-2} \mathrm{nM}$ by dissolving fluorescein disodium in the buffer. A cobalt solution was prepared by dissolving $47.1 \mathrm{mg}$ of cobalt (II) chloride hexahydrate and $60 \mathrm{mg}$ picolinic acid in $30 \mathrm{ml}$ of distilled water. A $1.1 \mathrm{M}$ hydrogen peroxide solution was prepared by diluting $30 \%$ $\mathrm{H}_{2} \mathrm{O}_{2}$ solution $(8.8 \mathrm{M})$ with distilled water. Gallic acid standard solutions were prepared at concentrations of 800,600 , 400, 200 and $100 \mu \mathrm{M}$ by dissolving gallic acid in the buffer.

Measurement of hydrophilic-HORAC. The hydrophilicHORAC value was obtained by measuring the hydroxyl radical elimination activity of hydroxyl radicals generated from Fenton's reaction [cobalt (II) chloride hexahydrate added to hydrogen peroxide reaction mixture], and measuring the time lapse degradation of fluorescein (i.e., the rate of decrease in the intensity of fluorescence). The HORAC assay was performed in a 96-well multilabel microplate reader (Mithras LB940, Berthold Technologies GmbH \& Co. KG, Germany) as described by Ou et al (14). Briefly, $20 \mu 1$ each of sample buffer (obtained by appropriate dilution with assay buffer), various concentrations of gallic acid standard solution (for construction of a standard curve) or blank buffer (as a control) were placed in individual wells of a 96-well transparent microplate (Sanplatec Corp., Osaka, Japan). Fluorescein working solution $(270 \mu \mathrm{l})$ was added and the well was agitated at $37^{\circ} \mathrm{C}$ for $10 \mathrm{~min}$. Subsequently, $10 \mu 1 \mathrm{H}_{2} \mathrm{O}_{2}$ and $10 \mu \mathrm{l}$ cobalt (II) chloride solutions were continuously added to each of the wells to initiate the reaction. The total volume of each reaction solution was $310 \mu \mathrm{l}$. The fluorescence intensity [485 nm (excitation)/535 nm (emission)] was then measured every $1 \mathrm{~min}$ for $65 \mathrm{~min}$ at $37^{\circ} \mathrm{C}$. As the reaction progressed, fluorescein was consumed and the fluorescence intensity decreased. The inhibition of fluorescence decay indicated the presence of an antioxidant.

Typical HORAC assay kinetic curves in the presence of different concentrations of gallic acid were determined. HORAC values were then determined.

The area under the kinetic curve (AUC) of the standards and samples was calculated as follows:

AUC $=\left(0.5+f_{1 \min } / f_{0 \min }+\mathrm{f}_{2 \min } / \mathrm{f}_{0 \min }+\mathrm{f}_{3 \min } / \mathrm{f}_{0 \min } \ldots \ldots+0.5 \mathrm{x}\right.$ $\left(\mathrm{f}_{65 \min } / \mathrm{f}_{0 \min }\right),\left(\mathrm{f}_{\mathrm{xmin}}=\right.$ fluorescence reading at cycle $\left.\mathrm{x} \mathrm{min}\right)$.

The standard regression line was obtained by plotting the gallic acid concentration against the net $\mathrm{AUC}_{\text {gallic acid }}$ of each concentration:

Net $\mathrm{AUC}_{\text {gallic acid }}=\mathrm{AUC}_{\text {gallic acid }}-\mathrm{AUC}_{\text {control }}$, Net $\mathrm{AUC}_{\text {sample }}=$ $\mathrm{AUC}_{\text {sample }}-\mathrm{AUC}_{\text {control }}\left(\mathrm{AUC}_{\text {gallic acid }}, \mathrm{AUC}\right.$ in the presence of gallic acid; $\mathrm{AUC}_{\text {control }}$, $\mathrm{AUC}$ with blank control; $\mathrm{AUC}_{\text {sample }}$, AUC with sample buffer).

The horizontal axis was regarded as the net $\mathrm{AUC}_{\text {gallic acid }}$ and the vertical axis as the concentration of gallic acid.

The equation of $Y=a x+b$ was derived from the above data, and the values for $\mathrm{a}$ and $\mathrm{b}$ were obtained.

The final HORAC values of the samples were calculated using the equation:

HORAC value $[\mu \mathrm{mol}$ gallic acid equivalent $(\mathrm{GAE}) / 100 \mathrm{ml}]=$ [a x (net $\mathrm{AUC}_{\text {sample }}$ )] x 100/[sample], 


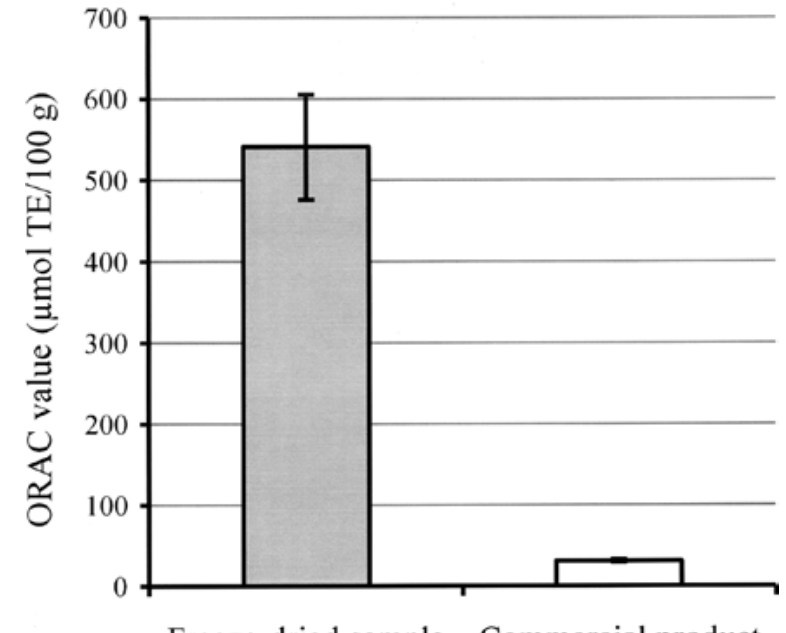

Freeze-dried sample Commercial product

Figure 1. Hydrophilic-ORAC values [ $\mu \mathrm{mol}$ trolox equivalent $(\mathrm{TE}) / 100 \mathrm{~g}]$ representing the peroxyl radical elimination capacity of freeze-dried powder and raw commercial samples of the giant jellyfish $(n=3)$.

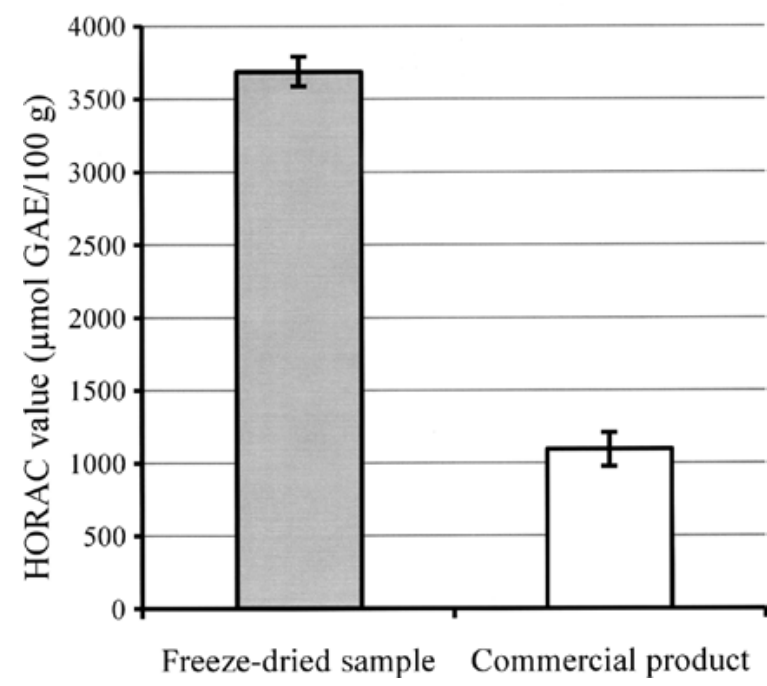

Figure 2. Hydrophilic-HORAC values [ $\mu \mathrm{mol}$ gallic acid equivalent $(\mathrm{GAE}) / 100 \mathrm{~g}$ ] representing the hydroxyl radical elimination capacity of freeze-dried powder and raw commercial samples of the giant jellyfish $(n=3)$.

where $[$ sample $]=$ the diluted concentration ratio of the sample .

Data were analyzed using Microsoft Excel.

Electron spin resonane analysis. ESR was performed as previously described $(6,7,13)$. Briefly, hydroxyl radical generation was first examined using the DMPO method and iron (II) sulfate with or without the Nemopilema nomurai samples. The addition of $8.8 \mathrm{mM} \mathrm{H}_{2} \mathrm{O}_{2}(50 \mu \mathrm{l})$ to the reaction mixture (320 $\mu \mathrm{l}$ ) was then used to initiate Fenton's reaction as depicted in the chemical equation: $\mathrm{Fe}^{2+}+\mathrm{H}_{2} \mathrm{O}_{2} \rightarrow \mathrm{Fe}^{3+}+\cdot \mathrm{OH}+\mathrm{OH}^{-}$. After $1 \mathrm{~min}$ of hydroxyl radical generation, spin adduct DMPO-OH was measured using an ESR spectrometer (JES-FR30; JEOL Ltd., Tokyo, Japan). ESR measurement conditions were: output, $4 \mathrm{~mW}(9.4 \mathrm{GHz})$; magnetic field,

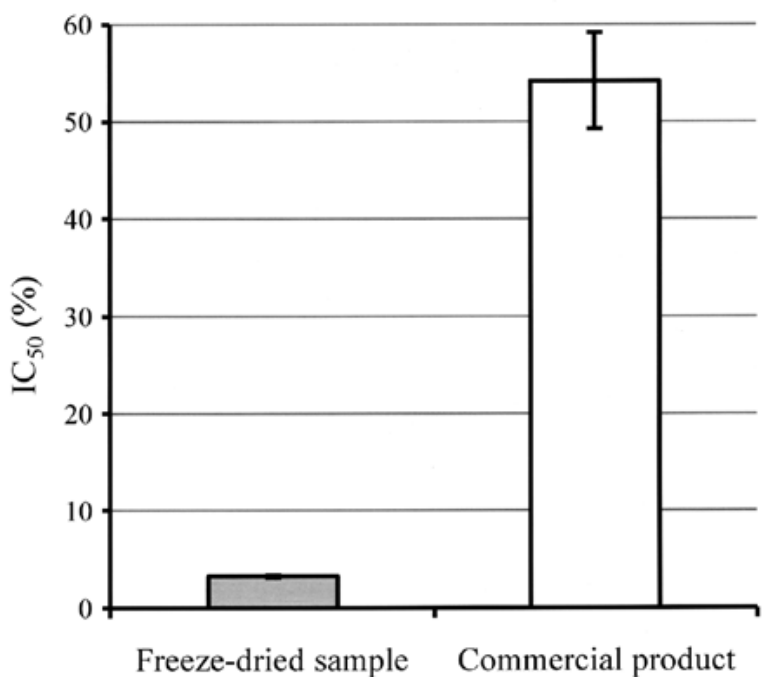

Figure 3. $\mathrm{IC}_{50}$ values (\%) representing the hydroxyl radical scavenging activities of freeze-dried powder and raw commercial samples of the giant jellyfish $(n=3)$.

$342,790 \pm 5 \mathrm{mT}$; modulation amplitude, 0,079 $\mathrm{mT}$; time constant, $0.1 \mathrm{sec}$; sweeping time, $1 \mathrm{~min}$; and amplification ratio, 32-125.

Calculation of $I C_{50}$ value of hydroxyl radical scavenging. $\mathrm{IC}_{50}$ values were defined as previously described $(6,7,13)$.

\section{Results and Discussion}

The results of hydrophilic-ORAC values of the giant jellyfish samples are shown in Fig. 1. The ORAC value of the freeze-dried sample was $540.9 \pm 64.8 \mu$ mol trolox equivalent (TE)/100 g, and the commercial sample was $30.8 \pm 1.5$, respectively. The low ORAC value of the commercial sample was due to the high body fluid content of the jellyfish in the sample. According to the USDA database, the ORAC value of the freeze-dried sample was similar to that for raw sweet corn (593 $\mu \mathrm{mol} \mathrm{TE} / 100 \mathrm{~g}$ ), raw cabbage (508), raw celery (512) and raw pumpkin (414).

On the other hand, for the first time, we reported the hydrophilic-HORAC value for a fishery product in this study. The results of HORAC values of the giant jellyfish samples are shown in Fig. 2. The HORAC value of the freeze-dried sample was 3,687.4 $\pm 100.9 \mu \mathrm{mol} \mathrm{GAE} / 100 \mathrm{~g}$, and the commercial sample was $1,093.4 \pm 116.8$. Since a database for HORAC values has yet to be established, we cannot currently assess whether or not the hydroxyl radical elimination activity of the freeze-dried powder sample of the giant jellyfish is high. Therefore, the hydroxyl radical scavenging activity of the freeze-dried powder sample was measured using the ESR method. From the results shown in Fig. 3 , the $\mathrm{IC}_{50}$ values of hydroxyl radical scavenging activity were found to be $3.27 \pm 0.12$ and $54.20 \pm 4.94 \%$ for $0.2 \mathrm{~g} / \mathrm{ml}$ freeze-dried powder and raw commercial sample solutions, respectively. We observed that the $\mathrm{IC}_{50}$ value of sandfish sauce obtained using the ESR method was $4.16 \%$, and the ratio of DNA protection (\%) was $60.1 \%$ (15). In addition, we noted that the average of $\mathrm{IC}_{50}$ values of raw and processed sea urchin foods obtained 
using the ESR method was $4.14 \%$, and that the average of $\mathrm{IC}_{50}$ values of peroxyl radical scavenging activity obtained via the chemiluminescence method was $0.33 \%$ (13). The $\mathrm{IC}_{50}$ value of $3.27 \%$ indicated a high hydroxyl radical scavenging activity. Therefore, it can be concluded that the HORAC value of 3,687.4 $\mu \mathrm{mol}$ GAE/100 g suggests a high hydroxyl radical elimination activity.

In their study, Yu et al reported that the protein from the tentacles of the jellyfish Rhopilema esculentum indicated the scavenging activities of superoxide anion and hydroxyl radical (16). Zhuang et al reported that the collagen from Rhopilema esculentum alleviated the UV-induced abnormal changes of antioxidative indicators, including superoxide dismutase, glutathione peroxidase and catalase activities, on mouse skin (17). Although the Nemopilema nomurai jellyfish stings workers harvesting the medusae and induces cutaneous symptoms in humans, there is no significant incidence of symptoms by ingestion (18). Kim et al observed that the venom of the giant jellyfish produced a functional cardiac depression in mice (19). Recently, Imamichi and Yokoyama reported the purification, characterization and cDNA cloning of a novel lectin, sugar-binding protein, stemming from this jellyfish (20). Lectins are highly specific for their sugar moieties and play a role in the biological recognition phenomena involving cells and proteins.

We attempted the practical usage of freeze-dried Nemopilema nomurai powder as a cooking ingredient in Japanese Udon noodles, including replacing salt with the powder obtained from the giant jellyfish (3). It is common practice in Japan that Udon noodles be made from wheat, salt and water, where salt is added to alter the gluten structure and increase the viscoelasticity of the noodles. Since the giant jellyfish is directly frozen with its body fluid, the freeze-dried powder was found to be relatively salty. Consequently, this freeze-dried giant jellyfish powder may be used as a functional salt for human health benefits.

\section{Acknowledgements}

The authors are grateful to Professor Tsuneo Shiba (Director of Department of Food Science and Technology, National Fisheries University) for his kind discussion, and to Mr. Takahisa Isono (Kyushu Maruichi Food Co., Ltd.) for providing us with the edible raw commercial giant jellyfish similar to Nemopilema nomurai. This study was performed in part by the project research of Research Institute of Domestic Science, Tokyo Kasei University between 2007 and 2009.

\section{References}

1. Dong Z, Liu D and Keesing JK: Jellyfish blooms in China: dominant species, causes and consequences. Mar Pollut Bull 60 954-963, 2010

2. Kang C, Munawir A, Cha M, et al: Cytotoxicity and hemolytic activity of jellyfish Nemopilema nomurai (Scyphozoa: Rhizostomeae) venom. Comp Biochem Physiol C Toxicol Phamacol 150: 85-90, 2009.

3. Nagatsuka N, Harada K, Ueno S and Nagao K: Practical usage of giant jellyfish as cooking foods and measurement of its antioxidative activity. Bulletin of Research Institute of Domestic Science, Tokyo Kasei University (in Japanese) 33: 25-29, 2010.
4. Okazaki E: III-4. Food utilization of the giant jellyfish Nemopilema nomurai. Nippon Suisan Gakkaishi (in Japanese) 71: 993-994, 2005.

5. Harada K, Okano C, Kadoguchi H, Okubo Y, Ando M, Kitao S and Tamura Y: Peroxyl radical scavenging capability of fish sauces measured by the chemiluminescence method. Int $\mathrm{J}$ Mol Med 12: 621-625, 2003.

6. Nagatsuka N, Harada K, Ando M and Nagao K: Measurement of the radical scavenging activity of chicken jelly soup, a part of the medicated diet, 'Yakuzen', made from gelatin gel food 'Nikogori', using chemiluminescence and electron spin resonance methods. Int J Mol Med 18: 107-111, 2006.

7. Harada K, Maeda M, Hasegawa Y, Tokunaga T, Tamura Y and Koizumi T: Antioxidant activity of fish sauces including puffer (Lagocephalus wheeleri) fish sauce measured by the oxygen radical absorbance capacity method. Mol Med Rep 3: 663-668, 2010.

8. Cao G, Alessio HM and Cutler RG: Oxygen-radical absorbance capacity assay for antioxidants. Free Radic Biol Med 14: 303-311, 1993.

9. Cao G, Verdon CP, Wu AH, Wang H and Prior RL: Automated assay of oxygen radical absorbance capacity with the COBAS FARA II. Clin Chem 41: 1738-1744, 1995.

10. USDA Database for the Oxygen Radical Absorbance Capacity (ORAC) of Selected Foods, Release 2. U.S. Department of Agriculture, Agricultural Research Service, Beltsville Human Nutrition Research Center, Nutrition Data Laboratory, Beltsville, Maryland, pp1-46, 2010.

11. Schauss AG, Wu X, Prior RL, et al: Antioxidant capacity and other bioactivities of the freeze-dried Amazonian palm berry, Euterpe oleraceae Mart, (acai). J Agric Food Chem 54: 8604-8610, 2006.

12. Miller MJ, Bobrowski P, Shukla M, Gupta K and Haqqi TM: Chondroprotective effects of a proanthocyanidin rich Amazonian genonutrient reflects direct inhibition of matrix metalloproteinases and upregulation of IGF-1 production by human chondrocytes. J Inflamm: Aug 14, 2007, doi: 10.1186/1476-9255-4-16 (E-pub ahead of print).

13. Maeda T, Komoda H, Tsujimura A, et al: Peroxyl and hydroxyl radical scavenging activities of raw and processed sea urchins measured by chemiluminescence and electron spin resonance methods. J Clin Biochem Nutr 43: S567-S570, 2008.

14. Ou B, Hampsch-Woodill M, Flanagan J, Deemer EK, Prior RL and Huang D: Novel fluorometric for hydroxyl radical prevention capacity using fluorescein as the probe. J Agric Food Chem 50: 2772-2777, 2002.

15. Harada K, Makino Y, Yamauchi T, et al: Efficacy of puffer fish (Takifugu rubripes) sauce in reducing hydroxyl radical damage to DNA assessed using the apurinic/apyrimidinic site method. Int J Mol Med 20: 309-314, 2007.

16. Yu H, Liu X, Xing R, Liu S, Li C and Li P: Radical scavenging activity of protein from tentacles of jellyfish Rhopilema esculentum. Bioorg Med Chem Lett 15: 2659-2664, 2005.

17. Zhuang Y, Hou H, Zhao X, Zhang Z and Li B: Effects of collagen and collagen hydrolysate from jellyfish (Rhopilema esculentum) on mice skin photoaging induced by UV irradiation. J Food Sci 74: H183-H188, 2009.

18. Kawahara M, Uye S, Burnett J and Mianzan H: Sting of edible jellyfish (Rhopilema hispidum, Rhopilema esculentum and Nemopilema nomurai) in Japanese waters. Toxicon 48: 713-716, 2006.

19. Kim E, Lee S, Kim JS, Yoon WD, Lim D, Hart AL and Hodgson WC: Cardiovascular effects of Nemopilema nomurai (Scyphozoa: Rhizostomeae) jellyfish venom in rats. Toxicol Lett 167: 205-211, 2006.

20. Imamichi Y and Yokoyama Y: Purification, characterization and cDNA cloning of a novel lectin from the jellyfish Nemopilema nomurai. Comp Biochem Physiol B Biochem Mol Biol 156: 12-18, 2010. 